\title{
Enhancement of piperlongumine chemosensitivity by silencing heme oxygenase-1 expression in cholangiocarcinoma cell lines
}

\author{
CHUTIMA TALABNIN ${ }^{1}$, KRAJANG TALABNIN ${ }^{2}$ and SOPIT WONGKHAM $^{3,4}$ \\ ${ }^{1}$ School of Chemistry, Institute of Science, Suranaree University of Technology; ${ }^{2}$ School of Pathology, Institute \\ of Science, Suranaree University of Technology, Nakhon Ratchasima 30000; ${ }^{3}$ Department of Biochemistry and \\ ${ }^{4}$ Cholangiocarcinoma Research Institute, Faculty of Medicine, Khon Kaen University, Khon Kaen 40002, Thailand
}

Received December 4, 2019; Accepted June 2, 2020

DOI: $10.3892 / \mathrm{ol} .2020 .11784$

\begin{abstract}
Piperlongumine (PL) produces reactive oxygen species (ROS) and induces G2/M-phase arrest in cholangiocarcinoma (CCA) cells via the JNK/ERK pathway. A differential response to PL was observed among all CCA cell lines However, the underlying mechanisms have remained to be fully elucidated. The aim of the present study was to investigate the molecular mechanisms of PL-induced heme oxygenase-1 (HO-1) expression in CCA cell lines. The anti-proliferative action of PL in the CCA cell lines KKU-100 and KKU-213A was analyzed using sulforhodamine B assays. Reverse transcription-quantitative PCR and western blot analyses were used to examine mRNA and protein expression. HO-1 inhibition was achieved using the chemical inhibitor zinc protophoryn or specific small interfering RNA to HO-1. Intracellular ROS was detected using a 2',7'-dichlorodihydrofluorescein diacetate fluorescence assay. High expression of phase-II detoxification enzymes, including NADPH quinone oxidoreductase-1, heme oxygenase-1, superoxide dismutases and aldo-keto reductase 1 subunits $\mathrm{C}-1$ and 3 , were detected in the KKU-100 cell line. Of the CCA cell lines tested, KKU-100 was the least sensitive to PL. Dose-dependent upregulation of HO-1 expression via PI3K/Akt activation was detected in PL-treated CCA cells. Inhibition of HO-1 eliminated the antioxidant defense mechanisms, leading to increased anti-cancer activity of PL in the CCA cell lines via an increase in intracellular ROS levels and apoptotic protein expression. These observations indicated that HO-1 inhibition had a chemosensitizing effect on CCA to PL.
\end{abstract}

Correspondence to: Dr Chutima Talabnin, School of Chemistry, Institute of Science, Suranaree University of Technology, 111 University Avenue, Nakhon Ratchasima 30000, Thailand

E-mail: chutima.sub@sut.ac.th

Key words: cholangiocarcinoma, piperlongumine, heme oxygenase-1, anti-oxidant capacity

\section{Introduction}

Cholangiocarcinoma (CCA) is a malignancy of bile duct epithelial cells. By 2002, CCA is the second most common type of primary liver cancer in most parts of the world (1). CCA has a poor prognosis, with a 5-year survival rate of 5-10\% worldwide between 2012 and 2013 (2,3). Surgical resection is the most effective treatment for CCA $(1,4)$. When resection is performed at an early stage, the 5-year survival rate increases to 25-30\% between 1997 and 2010 in Japan (4,5). In non-resectable, recurrent and metastatic CCA, various chemotherapeutic agents, including gemcitabine, cisplatin and oxaliplatin, have been used either alone or in combination (5). Resistance to chemotherapy, however, constrains the response rate to $0-40 \%$ and median survival to $2-12$ months between 1994 and 2002, worldwide (6-8).

The regulation of redox homeostasis is an essential factor in maintaining normal cellular functions and ensuring cell survival (9). High reactive oxygen species (ROS) levels in cancer cells are a consequence of alteration of several signaling pathways linked to tumorigenesis, including stimulation of cellular proliferation, as well as promotion of mutation and genetic instability $(9,10)$. Redox-modulating strategies are a potential treatment for patients with breast, ovarian, lung and pancreatic cancer that may enable therapeutic selectivity and help in overcoming drug resistance $(11,12)$. For instance, cancer cells with an increased level of ROS or decreased antioxidant capacity are more susceptible to oxidative stress-induced cell death (13). Certain anti-cancer agents, including arsenic trioxide, anthracyclines and cisplatin, have been demonstrated to act as ROS-generating agents that cause increased cellular ROS generation (10). These anti-cancer agents are candidates for evaluating the preferential targeting of cancer cells with increased ROS-induced stress $(9,10)$. The present study focused on piperlongumine (PL), a phytochemical that acts as an anti-cancer agent (14-16). PL induces redox dysregulation, selectively killing cancer cells (including CCA) with incremental increases in intracellular ROS (14). The increased sensitivity of cells to PL is associated with the degree of cell transformation (16). In addition, this was demonstrated in previous studies (15) on immortalized cholangiocytes and spontaneously immortalized fibroblasts $(15,16)$. PL-induced ROS generation is dependent on the activation of MAPKs, 
including JNK, ERK and p38 $(14,15)$. Various responses to PL have been reported, which may be due to differences in the underlying genetics of the antioxidant defense mechanism in each type of cancer cell (15).

Heme oxygenase-1 (HO-1), an inducible form of $\mathrm{HO}$, was the first rate-limiting enzyme discovered. In mammalian cells, HO-1 degrades cellular heme to release free iron, $\mathrm{CO}$ and biliverdin (17). HO-1 is frequently upregulated in numerous types of tumor, including prostate, renal, gastric, colon cancer and CCA (17-21). Upregulation of HO-1 is associated with tumor progression, including tumor growth, metastasis and chemoresistance $(20,21)$. Previous studies have demonstrated that the depletion of critical cytoprotective enzymes in cancer cells (particularly HO-1) enhanced the chemosensitivity of several anti-cancer agents, including gemcitabine, cisplatin and bortezomib $(20,22,23)$. The present study aimed to demonstrate that increased chemosensitivity of CCA may be achieved by a combination of anti-cancer agents, specifically PL targeting HO-1. The hypothesis was that HO-1 may be induced during PL treatment in CCA cell lines and that the suppression of HO-1 by a chemical inhibitor or specific small interfering (si)RNA may increase the level of intracellular ROS and chemosensitivity to PL.

\section{Materials and methods}

Materials. Cell culture reagents were from Gibco (Thermo Fisher Scientific, Inc.). PL, 2',7'-dichlorodihydrofluorescein diacetate (DCFH-DA; cat. no. D6883), trichloroacetic acid (cat. no. T0699), zinc-protoporphyrin IX (ZnPP; HO-1 inhibitor; cat. no. 691550) and sulforhodamine B (SRB; cat. no. 51402) were obtained from Sigma-Aldrich (Merck KGaA). Specific siRNA to HO-1 (siHO-1; cat. no. sc-35554) and non-targeted negative control siRNA (siCon; cat. no. sc-37007) were purchased from Santa Cruz Biotechnology, Inc. DharmaFect 1 siRNA transfection reagent (cat. no. T-2001-20) was purchased from GE Healthcare Dharmacon, Inc. Primary antibodies were obtained from Cell Signaling Technology, Inc., including total Akt (60 kDa) (cat. no. 4685S; 1:1,000), serine 473-phosphorylated Akt (pAkt; $60 \mathrm{kDa}$ ) (cat. no. 4060S; 1:1,000), poly(ADP-ribose) polymerase (PARP; $116 / 89 \mathrm{kDa}$ ) (cat. no. 9542; 1:1,000), lamin B1 (68 kDa) (cat. no. 13435; 1:1,000) and Bcl-2 (28 kDa) (cat. no. 4223; 1:1,000). Antibodies to HO-1 (32 kDa) (cat. no. sc-136960; 1:500), nuclear factor erythroid 2-related factor 2 (Nrf2; $110 \mathrm{kDa}$ ) (cat. no. sc-365949; 1:500), Bax (23 kDa) (cat. no. sc-526; 1:500), and $\beta$-actin (42 kDa) (cat. no. sc-47778; 1:2,000) were purchased from Santa Cruz Biotechnology, Inc. Mouse anti-rabbit IgG-horseradish peroxidase (HRP) (cat. no. NXA931; 1:2,000) and donkey anti-rabbit IgG-HRP (cat. no. NA934V; 1:2,000) secondary antibodies were obtained from Cytiva. The Luminata ${ }^{\mathrm{TM}}$ Forte Western HRP substrate detection reagents (cat. no. WBLUF0100) were purchased from Merck KGaA. The Superscript VILO ${ }^{\mathrm{TM}}$ cDNA synthesis kit (cat. no. 11754-050) was purchased from Invitrogen (Thermo Fisher Scientific, Inc.). The LightCycler $^{\circledR} 480$ RT-PCR System and the LightCycler ${ }^{\circledR} 480$ SYBR Green I master mix (cat. no. 04707516001) were from Roche Diagnostics GmbH. Wortmannin (cat. no. 9951S) was purchased from Cell Signaling Technology, Inc.

Cell culture and transfections. A total of 2 human CCA cell lines (KKU-100 and KKU-213A) had been established from tumor of patients with CCA with liver-fluke infection admitted to Srinagarind Hospital, Khon Kaen University (Khonkaen, Thailand), as described previously by Sripa et al $(24,25)$. Certificates of analyses were obtained from the Japanese Collection of Research Bioresources Cell Bank. Cells were cultured in Ham's F12 medium (cat. no. 21700-075; Gibco; Thermo Fisher Scientific, Inc.) supplemented with $1 \%$ penicillin-streptomycin (cat. no. 15140-122; Gibco; Thermo Fisher Scientific, Inc.) and 10\% FBS (cat. no. 10270-098; Gibco; Thermo Fisher Scientific, Inc.). Cells were incubated at $37^{\circ} \mathrm{C}$ in a humidified atmosphere containing $5 \% \mathrm{CO}_{2}$. Cells with $70-80 \%$ confluence at $24 \mathrm{~h}$ were trypsinized with $0.25 \%$ trypsin-EDTA and subcultured in the same media. Mycoplasma testing with MycoAlert mycoplasma detection kit (cat. no. LT07-418; Lonza Rockland, Inc.) was conducted for the cell lines used.

Inhibition of HO-1 was performed by transfecting HO-1 siRNA into the cell lines KKU-100 and KKU-213A. Cells were seeded into 6-well plates at a seeding density of $3-4 \times 10^{5}$ cells/well and incubated overnight. Cells were transfected with $10 \mu \mathrm{M}$ of siHO-1 or siCon using DharnaFect 1 siRNA transfection reagent for 24 or $48 \mathrm{~h}$. The transfection procedure was performed according to the manufacturer's protocol.

Drug treatments. A stock concentration of $50 \mathrm{mM}$ PL, $5 \mathrm{mM}$ $\mathrm{ZnPP}$ and $2 \mathrm{mM}$ wortmannin was prepared in DMSO and stored in aliquots at $-20^{\circ} \mathrm{C}$ until use. Various concentrations of PL $(0.01,0.1,1,10,25,50$ and $100 \mu \mathrm{M})$ or $\mathrm{ZnPP}(0,1,5$ and $10 \mu \mathrm{M})$ were diluted with cell culture media for subsequent experiments. The vehicle control was DMSO with $0.001 \%$ concentration used in the preparation of the PL, $\mathrm{ZnPP}$ or wortmannin working solutions. For the combination treatment of PL and ZnPP or PL and wortmannin, cells were pre-treated with $\mathrm{ZnPP}(0,1,2.5,5.0,10,25,50$ and $100 \mu \mathrm{M})$ for $3 \mathrm{~h}$ or wortmannin $(1,2$ and $5 \mu \mathrm{M})$ for $2 \mathrm{~h}$. Then, ZnPP or wortmannin were removed and cultured for $24 \mathrm{~h}$ or were removed prior to being treated with various concentrations of PL $(0,0.01,0.1,1,10,25,50$ and $100 \mu \mathrm{M}$; or 0,10 and $20 \mu \mathrm{M}$, respectively) for $24 \mathrm{~h}$. For the PL treatment after transfection with siHO-1 or siCon, transfected cells were seeded at $5 \times 10^{3}$ cells per well into a 96 well plate. At $24 \mathrm{~h}$ after seeding, cells were treated with a range of concentrations of PL from 0,5, 10 and $20 \mu \mathrm{M}$ for $24-\mathrm{h}$. Treated cells were subsequently tested for cell viability at $48 \mathrm{~h}$, intracellular ROS and assessed by reverse transcription-quantitative PCR (RT-qPCR) and western blot analysis.

Cell viability. KKU-100 and KKU-213A cells were seed at $5 \times 10^{3}$ cells per well into a 96-well plate. At $24 \mathrm{~h}$ after seeding, the CCA cell lines were treated with drug as aforementioned and incubated for $24 \mathrm{~h}$. Cell viability was measured using an SRB assay capable of determining cell density based on the measurement of cellular protein content, performed according to Voigt with slight modifications (26). In brief, cells were fixed with $10 \%$ trichloroacetic acid for overnight, washed 5 times with distilled water and stained with $0.4 \%$ SRB in $1 \%$ acetic acid for $30 \mathrm{~min}$. Plates were washed 5 times with $1 \%$ acetic acid, air-dried and then solubilized the protein-bound dye with $100 \mu \mathrm{l}$ of unbuffered $10 \mathrm{mM}$ Tris solution $(\mathrm{pH}=10)$. The absorbance was measured at $564 \mathrm{~nm}$ using a microplate 
Table I. Sequences of the primers used for reverse transcription-quantitative PCR.

\begin{tabular}{lll}
\hline Gene & \multicolumn{1}{c}{ Forward (5'-3') } & \multicolumn{1}{c}{ Reverse $\left(5^{\prime}-3^{\prime}\right)$} \\
\hline Nrf2 & TACTCCCAGGTTGCCCACA & CATCTACAAACGGGAATGTCTGC \\
GCLM & GACAAAACACAGTTGGAACAGC & CAGTCAAATCTGGTGGCATC \\
GCLC & ATGCCATGGGATTTGGAAT & AGATATACTGCAGGCTTGGAATG \\
AKR1C1 & CATGCCTGTCCTGGGATTT & AGAATCAATATGGCGGAAGC \\
AKR1C3 & CATTGGGGTGTCAAACTTCA & CCGGTTGAAATACGGATGAC \\
HO-1 & CAACATCCAGCTCTTTGAGGA & GGGCAGAATCTTGCACTTTG \\
NQO1 & GATATTCCAGTTCCCCCTGC & TTCTTACTCCGGAAGGGTCC \\
TXN & GAGAGCAAGACTGCTTTTCA & CAGAGAGGGAATGAAAGAAAG \\
GSTP1 & TACACCAACTATGAGGCGGG & AGCGAAGGAGATCTGGTCTC \\
PARK7 2 & GTTGGCCAAGGGAGATGTTAC & AGCAACTCCCCTTTGGGTTC \\
$\beta$-actin & CGAGCTGGGATTAAGGTCA & CATATGGTCCCTCTTTTTTGC \\
\hline
\end{tabular}

Nrf2, nuclear factor erythroid 2-related factor 2; GCLM, $\gamma$-glutamylcysteine synthetase modifier subunit; GCLC, $\gamma$-glutamylcysteine synthetase catalytic subunit; AKR1C1 and 3, aldo-keto reductase 1 subunits C-1 and 3; HO-1, heme oxygenase-1; NQO1, NADPH quinone oxidoreductase-1; TXN, thioredoxin; GSTP1, glutathione S-transferase P1; SOD2, superoxide dismutase 2; PARK7, Parkinson disease protein 7 precursor.

reader (Bio-Rad Laboratories, Inc.). The cell viability was calculated as follows: Cell viability $(\%)=$ [optical density at $564 \mathrm{~nm}$ (OD564) in treatment wells]/(OD546 in control wells) $\mathrm{x} 100$. Each experiment was performed independently in triplicate. The half-maximal inhibitory concentration $\left(\mathrm{IC}_{50}\right)$ values were calculated using GraphPad Prism software (version 5.0; GraphPad Software, Inc.).

Measurement of intracellular accumulation of ROS. The production of intracellular ROS was detected using the DCFH-DA fluorescence assay, as previously described (10). In brief, KKU-100 cells were used for detecting ROS production. Thus, transfected KKU-100 cells $\left(2 \times 10^{5}\right.$ cells) were seeded into 6 -well plates and stored overnight for $30 \mathrm{~h}$ post-transfection. Cells were then treated with PL at 0, 10 and $20 \mu \mathrm{M}$ for $12 \mathrm{~h}$. Following treatment, the intracellular ROS assay was performed by incubation with $20 \mu \mathrm{M}$ of DCFH-DA in a humidified atmosphere with $5 \% \mathrm{CO}_{2}$ at $37^{\circ} \mathrm{C}$ for $30 \mathrm{~min}$. After washing with $1 \mathrm{X}$ PBS, cells were trypsinized and re-suspended in $1 \mathrm{X}$ PBS. The fluorescence intensity of the DCFH-DA was determined with a flow cytometer.

$R T-q P C R$. KKU-100 and KKU-213A cells that underwent drug treatments or transfection were harvested using TRIzol reagent for RNA preparation. In brief, total RNA was extracted using TRIzol reagent (cat. no. 15596; Invitrogen; Thermo Fisher Scientific, Inc.), according to the manufacturer's protocol. First-stand complementary (c)DNA was synthesized using a Superscript VILO ${ }^{\mathrm{TM}}$ cDNA synthesis kit (Invitrogen; Thermo Fisher Scientific, Inc.) with $2 \mu \mathrm{g}$ total RNA according to the manufacturer's protocol. The basal mRNA expression of 11 antioxidant-associated genes including nuclear factor erythroid 2-related factor 2 (Nrf2), NADPH quinone oxidoreductase-1 (NQO-1), heme oxygenase-1 (HO-1), superoxide dismutase 2 (SOD2), glutathione S-transferase P1 (GSTP1), aldo-keto reductase 1 subunits $\mathrm{C}-1$ and 3 (AKR1C1 and AKR1C3), $\gamma$-glutamylcysteine synthetase catalytic subunit (GCLC) and $\gamma$-glutamylcysteine synthetase modifier subunit (GCLM), Parkinson disease protein 7 precursor (PARK7), thioredoxin (TXN) was investigated in the two CCA cell lines, which have differential responses to PL; KKU-100 (CCA less sensitive to PL) and KKU-213A (CCA highly sensitive to PL). The sequences of the primers used are listed in Table I. Real-time PCR was performed using the LightCycler $^{\circledR} 480$ RT-PCR System and the LightCycler ${ }^{\circledR} 480$ SYBR-Green I master mix (Roche Diagnostics GmbH). The thermocycling conditions were as follows, according to the LightCycler 480 manufacturer's instructions: $95^{\circ} \mathrm{C}$ for $5 \mathrm{~min}$; $95^{\circ} \mathrm{C}$ for $10 \mathrm{sec}$, annealing at $60^{\circ} \mathrm{C}$ for $10 \mathrm{sec}$ and extension at $72^{\circ} \mathrm{C}$ for $10 \mathrm{sec}$, for 45 cycles. The specificity of each of the PCR products was confirmed via melting curve analysis. Relative mRNA expression was obtained following normalization to endogenous human $\beta$-actin and quantified using the $2^{-\Delta \Delta \mathrm{Cq}}$ methods (27).

Protein preparation. The cell lines KKU-100 and KKU-213A were cultured in 6 -well plates at $3-4 \times 10^{5}$ cells/well for $24 \mathrm{~h}$ at $37^{\circ} \mathrm{C}$ in a humidified atmosphere containing $5 \% \mathrm{CO}_{2}$. Cells were then treated with siRNA and/or various concentrations of $\operatorname{PL}(0,5,10$ or $20 \mu \mathrm{M})$ for $0,1,3,6$ or $24 \mathrm{~h}$. Following incubation, the whole-cell lysate was harvested as previously described by Thongsom et al (15). Nuclear and cytoplasmic fractions were extracted using a nuclear extraction kit (cat. no. 90498; Chemicon; Thermo Fisher Scientific, Inc.) according to the manufacturer's protocol. The whole-cell lysate and nuclear and cytoplasmic fractions were then tested for their respective protein concentrations using a Pierce ${ }^{\circledR}$ BCA protein assay kit (cat. no. 23225; Thermo Fisher Scientific, Inc.) according to the manufacturer's protocol.

SDS-PAGE and western blot analysis. Protein samples from the whole-cell lysate, nuclear or cytoplasmic fraction with a mass of $20 \mu \mathrm{g}$ were separated using $10 \%$ SDS-PAGE. The proteins were transferred to a nitrocellulose membrane and 


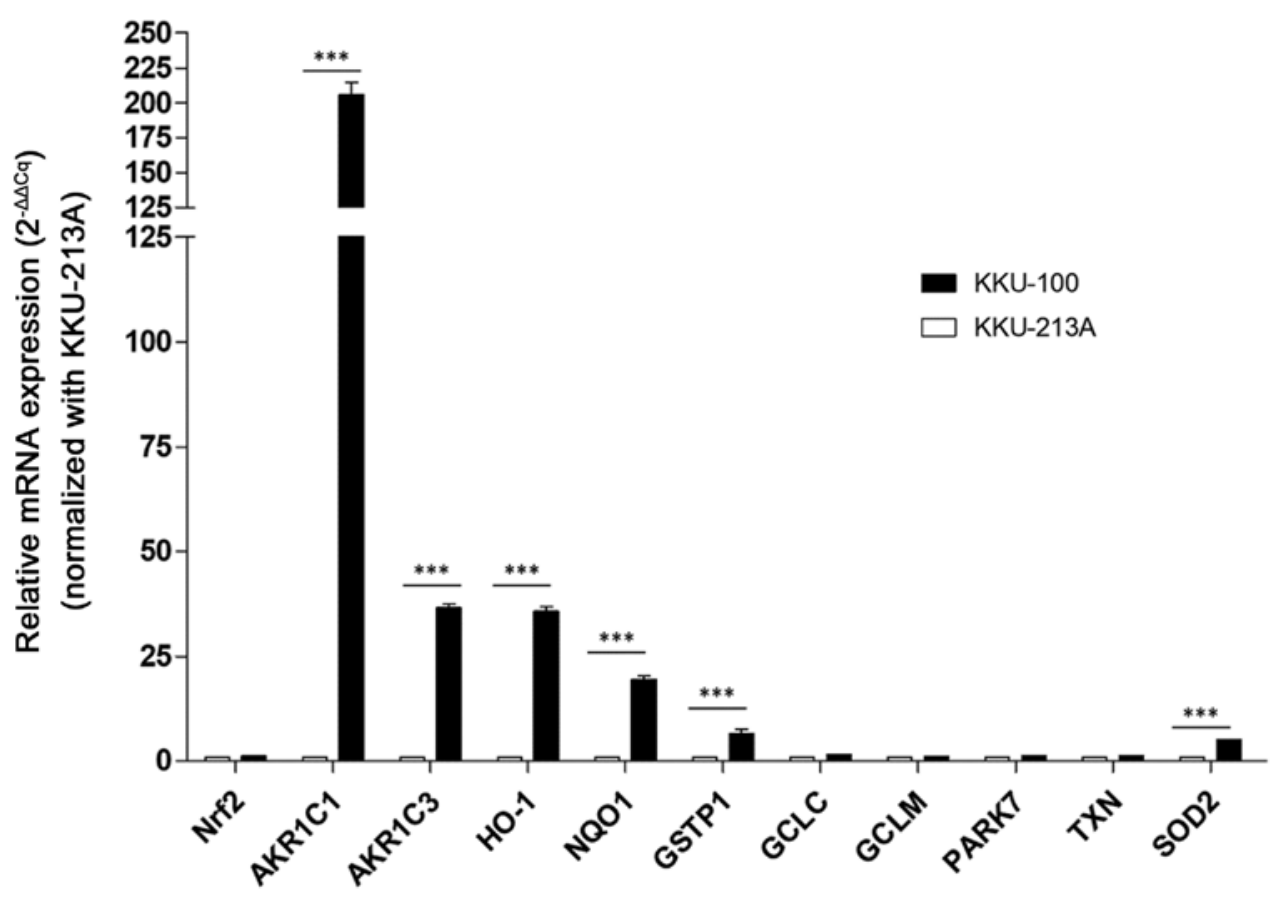

Figure 1. Antioxidant expression profiles in the cholangiocarcinoma KKU-100 and KKU-213A cell lines. mRNA expression of all antioxidant-associated genes was assessed using reverse transcription-quantitative PCR with normalization to $\beta$-actin used as the reference gene. mRNA expression of all genes was calculated using the $2^{-\Delta \Delta \mathrm{Cq}}$ method. Values are expressed as the mean \pm standard error of the mean of two independent experiments. ${ }^{* * *} \mathrm{P}<0.001$ vs. KKU-213A. $\mathrm{Nrf} 2$, nuclear factor erythroid 2-related factor 2; AKR1C1/3, aldo-keto reductase 1 subunits C-1/3; HO-1, heme oxygenase 1; NQO1, NADPH quinone oxidoreductase-1; GSTP1, glutathione S-transferase P1; GCLC, $\gamma$-glutamylcysteine synthetase catalytic subunit; GCLM, $\gamma$-glutamylcysteine synthetase modifier subunit; PARK7, Parkinson disease protein 7 precursor; TXN, thioredoxin; SOD2, superoxide dismutase 2.

blocked for $1 \mathrm{~h}$ with 5\% (w/v) skimmed milk in 1X PBS supplemented with $0.05 \%$ Tween-20 (PBST). The membranes were then incubated overnight at $4^{\circ} \mathrm{C}$ with primary antibodies at dilutions of 1:500 for HO-1, Bax and Nrf2 proteins; 1:1,000 for PARP, Bcl-2, pAkt (Ser473), total Akt and Lamin B1 proteins; or 1:2,000 for $\beta$-actin protein in PBST. The membranes were then incubated with HRP-conjugated secondary antibodies at a dilution of 1:2,000 for $1 \mathrm{~h}$ at room temperature. Luminata ${ }^{\mathrm{TM}}$ Forte Western HRP substrate (Merck KGaA) was applied for protein detection. The densities of the bands for HO-1, cleaved PARP, Bcl-2, Bax, Nrf2, Akt and pAkt were determined using Image $\mathrm{J}$ software version $1.52 \mathrm{v}$ (National Institutes of Health) and normalized to $\beta$-actin for whole cell lysate or normalized to lamin B1 for nuclear extracts. The respective ratio of each protein to $\beta$-actin, the ratio of $\mathrm{pAkt} / \mathrm{Akt}$, the ratio of cleaved $\mathrm{PARP} / \mathrm{Bcl}-2$ and ratio of $\mathrm{Bcl}-2 / \mathrm{Bax}$ were calculated.

Statistical analysis. All experiments were performed 2-3 times and the results are presented as the mean \pm standard error of the mean. Statistical analyses were performed using GraphPad Prism software (version 5.0; GraphPad Software, Inc.). The Student's t-test was used for between-group statistical analyses. One-way and two-way analysis of variance followed by Bonferroni's correction were applied for statistical analysis of multiple groups. $\mathrm{P}<0.05$ was considered to indicate a statistically significant difference.

\section{Results}

Expression levels of phase II detoxification enzymes and antioxidant proteins in CCA cell lines. Nrf2-mediated cytoprotective genes are thought to be a primary antioxidant defense mechanism in mammalian cells. The process eliminates harmful ROS or carcinogens, particularly phase II detoxification enzymes and antioxidant proteins (10). In the present study, basal mRNA expression of 11 antioxidant-associated genes was investigated in two CCA cell lines previously (15) indicated to have differential responses to PL: KKU-100 (CCA less sensitive to PL) and KKU-213A (CCA highly sensitive to PL). The results demonstrated that mRNA expression levels of phase II detoxification enzymes, including NADPH quinone oxidoreductase-1 (NQO-1), heme oxygease-1 (HO-1), superoxide dismutase 2 (SOD2), glutathione S-transferase P1 (GSTP1) and aldo-keto reductase 1 subunits C-1 and 3 (AKR1C1 and AKR1C3) were significantly higher in KKU-100 compared with KKU-213A (Fig. 1). Furthermore, the basal expression levels of Nrf2 and other Nrf2-mediated cytoprotective genes, including PARK7, thioredoxin TXN and particularly enzymes involved in glutathione synthesis, including GCLC and GCLM, were not significantly different. These results indicated that the mechanism of action for antioxidant defense may depend on the genetic background of each CCA cell line.

Induction of HO-1 expression following PL treatment. To evaluate the role of Nrf2-mediated cytoprotective genes in the responses to PL treatment, CCA cell lines were treated with $10 \mu \mathrm{M}$ PL or DMSO (control) for $6 \mathrm{~h}$. The respective mRNA expression of Nrf2-mediated cytoprotective genes (AKR1C1, AKR1C3, NQO-1, HO-1, GCLC and GCLM) was determined using RT-qPCR. The results revealed that the expression levels of HO-1, GCLC and GCLM were significantly increased in 
A

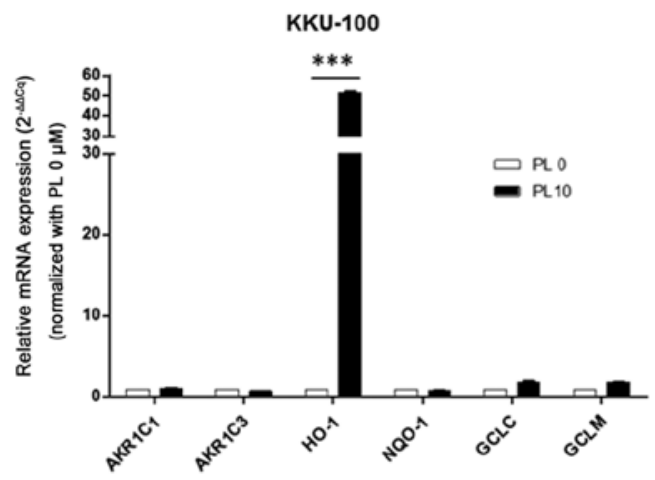

B
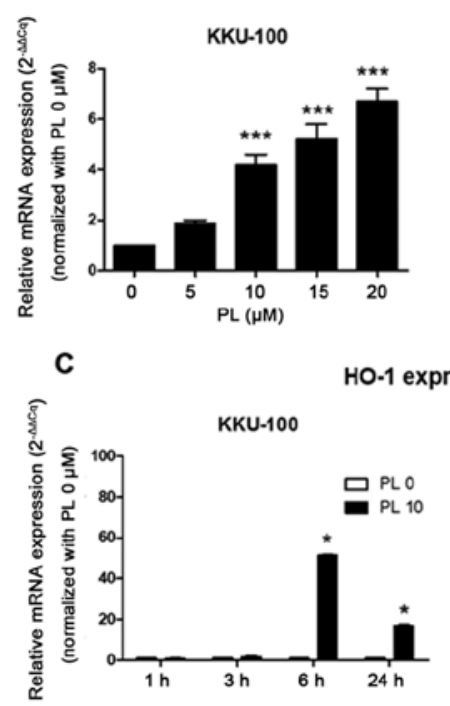

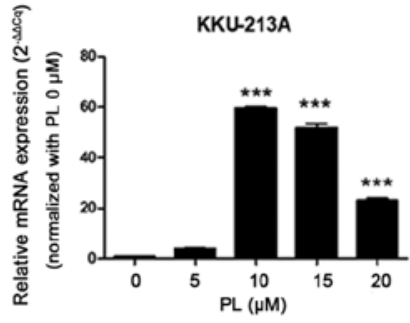

ession

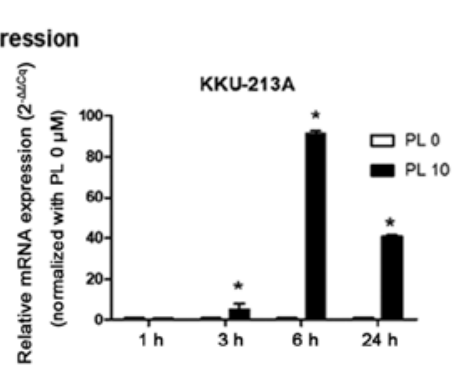

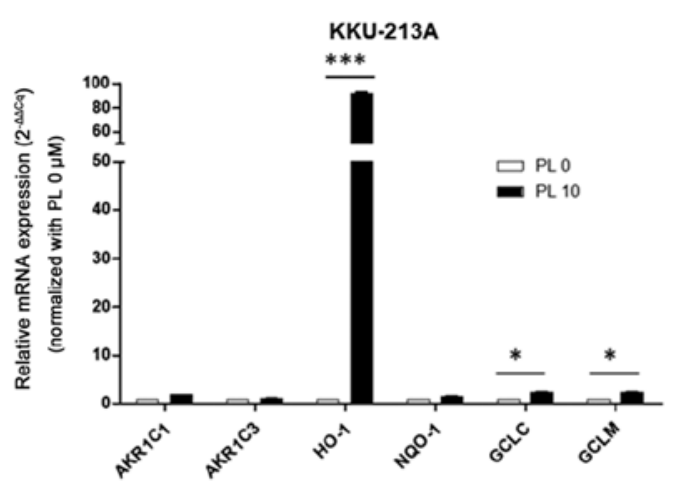
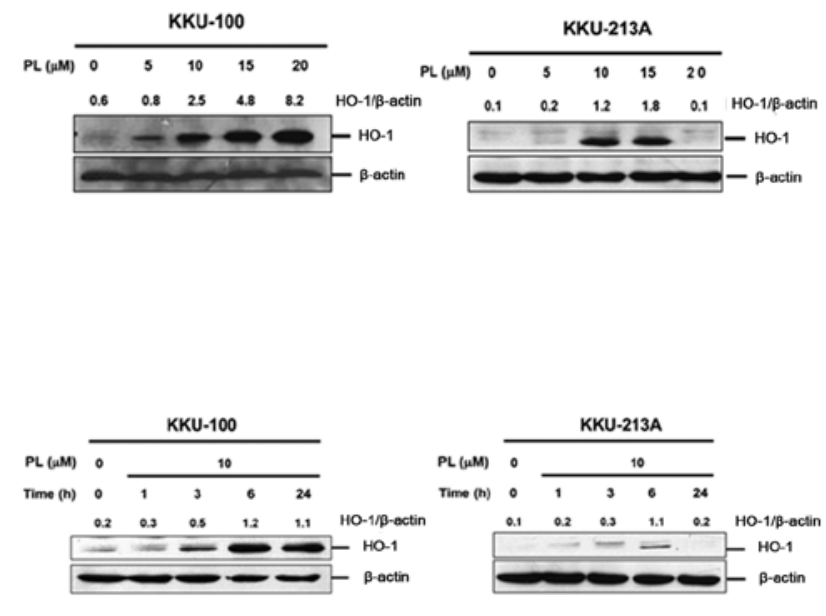

Figure 2. PL induces HO-1 expression in cholangiocarcinoma cell lines. (A) KKU-100 and KKU-213A were treated with PL at $10 \mu \mathrm{M}$ or DMSO (control) for $6 \mathrm{~h}$ and the mRNA expression of AKR1C1, AKR1C3, NQO1, HO-1, GGCL and GCLM was determined using RT-qPCR. Results were normalized using $\beta$-actin as the reference gene. Relative mRNA expression was calculated using the $2^{-\Delta \Delta C q}$ method. KKU-100 and KKU-213A treated with (B) PL (at $0,5,10,15$ or $20 \mu \mathrm{M}$ ) for $24 \mathrm{~h}$ or (C) $10 \mu \mathrm{M}$ PL for $0,1,3,6$ or $24 \mathrm{~h}$. The relative mRNA and protein expression of HO-1 was determined using RT-qPCR ( $\left.2^{-\Delta \Delta C q}\right)$ and western blot analysis, respectively. Values are expressed as the mean \pm standard error of the mean of 3 independent experiments. Protein expression is presented as the mean of two independent experiments. ${ }^{*} \mathrm{P}<0.05$ and ${ }^{* * *} \mathrm{P}<0.001$ vs. PL $0 \mu \mathrm{M}$. PL, piperlongumine; AKR1C1 and 3, aldo-keto reductase 1 subunits $\mathrm{C}-1$ and 3 ; NQO1, NADPH quinone oxidoreductase-1; HO-1, heme oxygenase 1; GCLC, $\gamma$-glutamylcysteine synthetase catalytic subunit; GCLM, $\gamma$-glutamylcysteine synthetase modifier subunit; RT-qPCR, reverse transcription-quantitative PCR.

KKU-213A; however, only HO-1 expression was significantly increased in KKU-100 following PL treatment. Therefore, HO-1 was the only Nrf2-mediated cytoprotective gene whose expression was increased in both CCA cell lines following PL treatment (Fig. 2A).

Subsequently, HO-1 expression in the CCA cell lines in the presence of PL at various concentrations $(0,5,10,15$ and $20 \mu \mathrm{M})$ for $24 \mathrm{~h}$ or $10 \mu \mathrm{M}$ of PL for various lengths of time $(0,1,3,6$ and $24 \mathrm{~h}$ ) was examined. The expression of HO-1 at the mRNA and protein levels in KKU-100 and KKU-213A cell lines following PL treatment was altered in a dose-dependent manner; however, while HO-1 expression increased in KKU-100, expression of HO-1 in KKU-213A peaked at $10 \mu \mathrm{M}$ and subsequently decreased (Fig. 2B). In addition, the induction of HO-1 expression increased in response to PL treatment for different durations, particularly at $6 \mathrm{~h}$ and then declined following $24 \mathrm{~h}$ of PL treatment (Fig. 2C). These results indicated that PL preferentially triggered the induction of HO- 1 expression in CCA cell lines and that the activation of HO-1 expression may be an early antioxidant defense in response to PL treatment.
HO-1 silencing promotes PL-induced CCA cell death by increasing ROS accumulation. Subsequently, it was investigated whether HO-1 acts as a key antioxidant defense for protecting from PL-mediated ROS generation in CCA cells. ZnPP, a chemical HO-1 inhibitor, was utilized to inhibit HO-1 activity in KKU-100 and KKU-213A. The effect of HO-1 inhibition was then evaluated with respect to PL-induced cytotoxicity. As $\mathrm{ZnPP}$ acts as an enzymatic substrate of $\mathrm{HO}-1$, it competes with heme for HO-1, which leads to decreasing levels of $\mathrm{CO}$ and bilirubin that act as an antioxidant defense (28). As previous studies have demonstrated that $\mathrm{ZnPP}$ is a cytotoxic agent and exhibits anti-tumor activity (29-31), the cytotoxicity of $\mathrm{ZnPP}$ was examined. The results demonstrated that $>10 \mu \mathrm{M}$ ZnPP suppressed CCA cell viability, with $<50 \%$ viable cells remaining in the KKU-100 following $3 \mathrm{~h}$ of treatment, whereas $<50 \%$ cell viability in the KKU-213A was observed at $\mathrm{ZnPP}>25 \mu \mathrm{M}$ (Fig. 3A). Subsequently, cell lines were pre-treated with $0-10 \mu \mathrm{M} \mathrm{ZnPP}$ for $3 \mathrm{~h}$ in combination with $0-100 \mu \mathrm{M}$ PL treatment to examine the effect of PL during HO-1 suppression by $\mathrm{ZnPP}$. The respective $\mathrm{IC}_{50}$ values of PL 
A

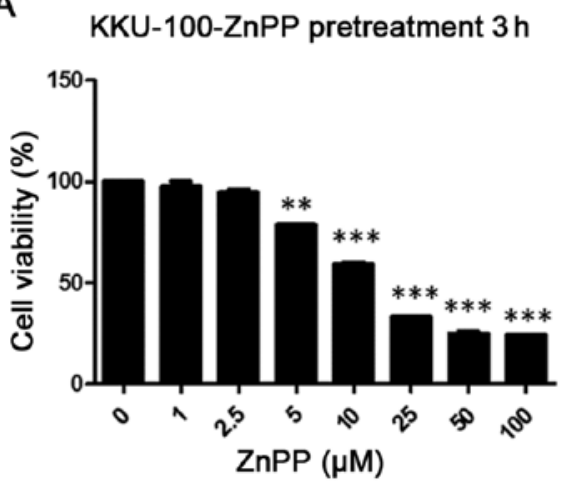

KKU-213A-ZnPP pretreatment $3 \mathrm{~h}$

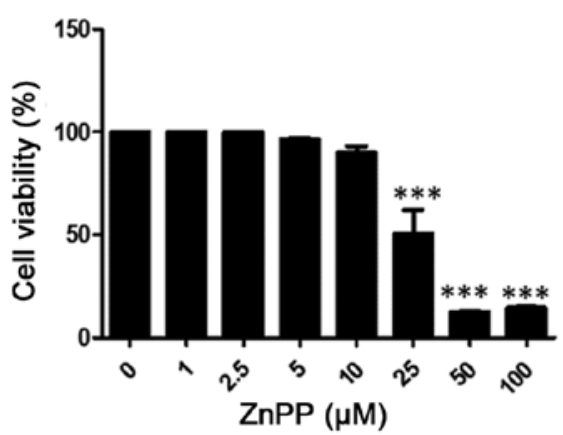

B

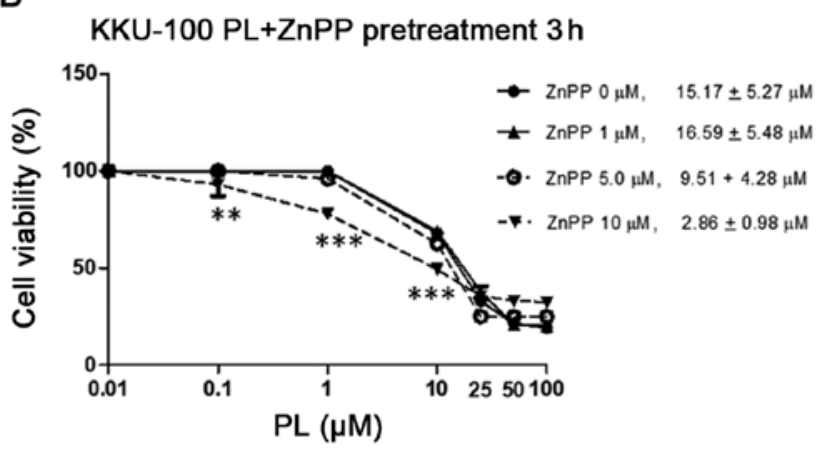

KKU-213A-PL+ZnPP pretreatment $3 \mathrm{~h}$

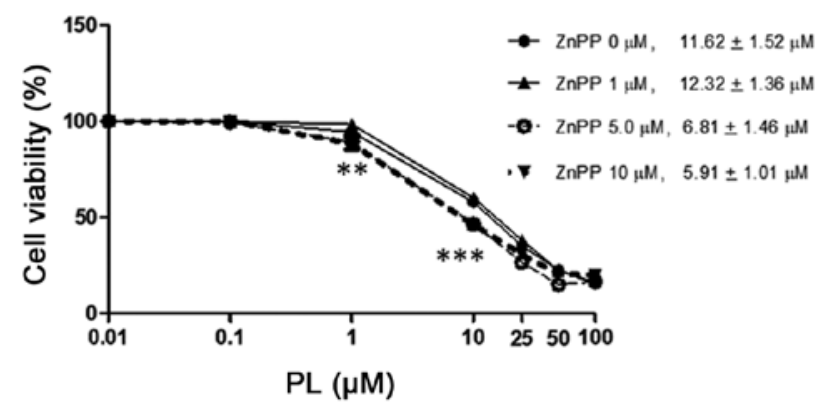

Figure 3. Inhibition of heme oxygenase 1 by ZnPP increases the anti-tumor activity of PL in cholangiocarcinoma cell lines. (A) KKU-100 and KKU-213A were pre-treated with ZnPP at various concentrations for $3 \mathrm{~h}$ then cultured without ZnPP for $24 \mathrm{~h}$. (B) KKU-100 and KKU-213A were pre-treated with ZnPP at 0 , 1, 5 and $10 \mu \mathrm{M}$ for $3 \mathrm{~h}$. ZnPP was then removed and cells were treated with $10 \mu \mathrm{M}$ PL for $24 \mathrm{~h}$. Cell viability was measured using sulforhodamine B assays. Values are expressed as the mean \pm standard error of the mean of 3 independent experiments. ${ }^{* *} \mathrm{P}<0.01$ and ${ }^{* * *} \mathrm{P}<0.001$ vs. ZnPP $0 \mu \mathrm{M}$. ZnPP, zinc-protoporphyrin IX; PL, piperlongumine.

were reduced compared with the combination of $\mathrm{ZnPP}$ at 5 and $10 \mu \mathrm{M}$ in both KKU-100 and KKU-213A cells (Fig. 3B).

Following this, HO-1 knockdown was used to elucidate the role of HO-1 in the sensitivity of CCA cell lines to PL. The mRNA expression levels of HO-1 in both KKU-100 and KKU-213A were significantly decreased following transfection with HO-1 siRNA at the time-points of 24 and 48 h (Fig. 4A); however, suppression of HO-1 was not influenced by CCA cell growth (Fig. 4B). The knockdown of HO-1 significantly enhanced the anti-tumor activity of PL in a dose-dependent manner for both KKU-100 and KKU-213A (Fig. 4C). In addition, the combination of HO-1-silencing with PL treatment at $20 \mu \mathrm{M}$ resulted in a significant increase in the accumulation of intracellular ROS. This result indicated that the effective dose of PL to induce ROS accumulation in KKU-100 was $20 \mu \mathrm{M}$ (Fig. 4D). Response to combination treatments in KKU-100 at $12 \mathrm{~h}$ was detectable via western blot analysis, which confirmed the upregulation of apoptotic proteins (cleaved PARP) and downregulation of anti-apoptotic proteins (Bcl-2; Fig. 4E and F). Furthermore, the increase of PL-induced CCA apoptosis through HO-1 suppression was clearly demonstrated by a high ratio of cleaved PARP/Bcl-2 and a low ratio of $\mathrm{Bcl}-2 / \mathrm{Bax}$ (Fig. 4F). Therefore, inhibition of HO-1 promoted PL-mediated ROS generation, leading to PL-induced CCA cell apoptosis.

PL induces Nrf2-mediated HO-1 expression via activation of the PI3K/Akt pathway. Previous studies have reported that the
PI3K/Akt pathway acts as a survival signal against multiple apoptotic insults and is hypothesized to be a major upstream signaling event prior to the induction of Nrf2-mediated HO-1 expression $(32,33)$. Furthermore, Lee et al (34) demonstrated that PL directly binds to cysteine residues by a thiol modification within kelch-like ECH-associated protein (Keap1) and that this direct binding promoted nuclear translocation of Nrf2 and subsequent upregulation of HO-1 expression. The activation/phosphorylation of Akt in PL-treated CCA cell lines was determined in order to elucidate the role of PI3K/Akt in PL-induced Nrf2 activation and HO-1 expression. HO-1 and Akt phosphorylation increased in response to PL treatment (Fig. 5A). Nuclear Nrf2 was also observed in a dose-dependent manner (5, 10 and $20 \mu \mathrm{M}$; Fig 5A). To demonstrate the association between PI3K/Akt signaling and HO-1 expression, various concentrations (1, 2 and $5 \mu \mathrm{M})$ of wortmannin, a specific inhibitor of PI3K, were used to inhibit PI3K/Akt activation. The level of HO-1 expression was then determined via western blot analysis. Wortmannin significantly inhibited Akt phosphorylation and PL-induced HO-1 expression in CCA cells in a dose-dependent manner (Fig. 5B). These results indicated that PL-induced HO-1 expression is stimulated via Nrf2/PI3K/Akt activation.

\section{Discussion}

PL exerts an anti-tumor effect on various types of cancer, including glioblastoma, lung and CCA $(14,15,35)$. PL inhibits 
A

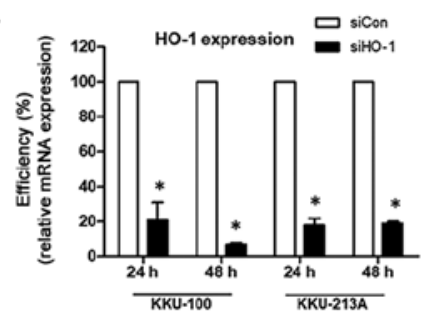

D
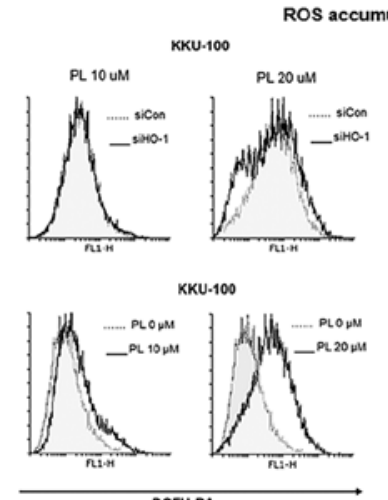

$\mathbf{F}$

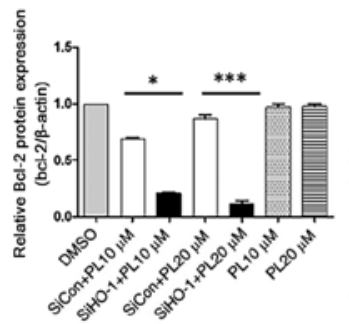

B

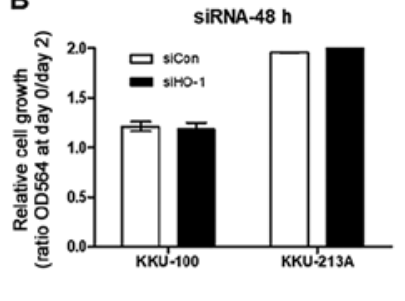

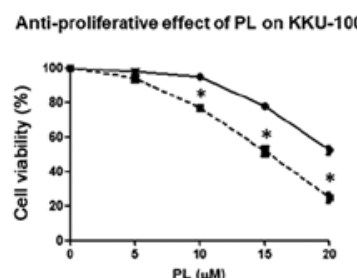

Anti-proliferative effect of PL on KKU-213A

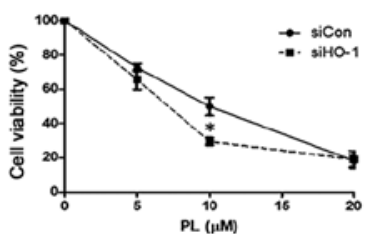

PL ( $\mu$ M) $0 \quad 10 \quad 10 \quad 20 \quad 20 \quad 10 \quad 20$

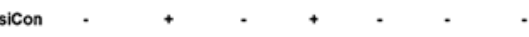
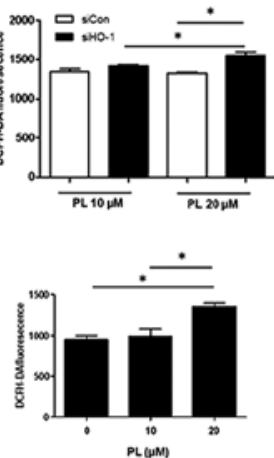

PL(UM)
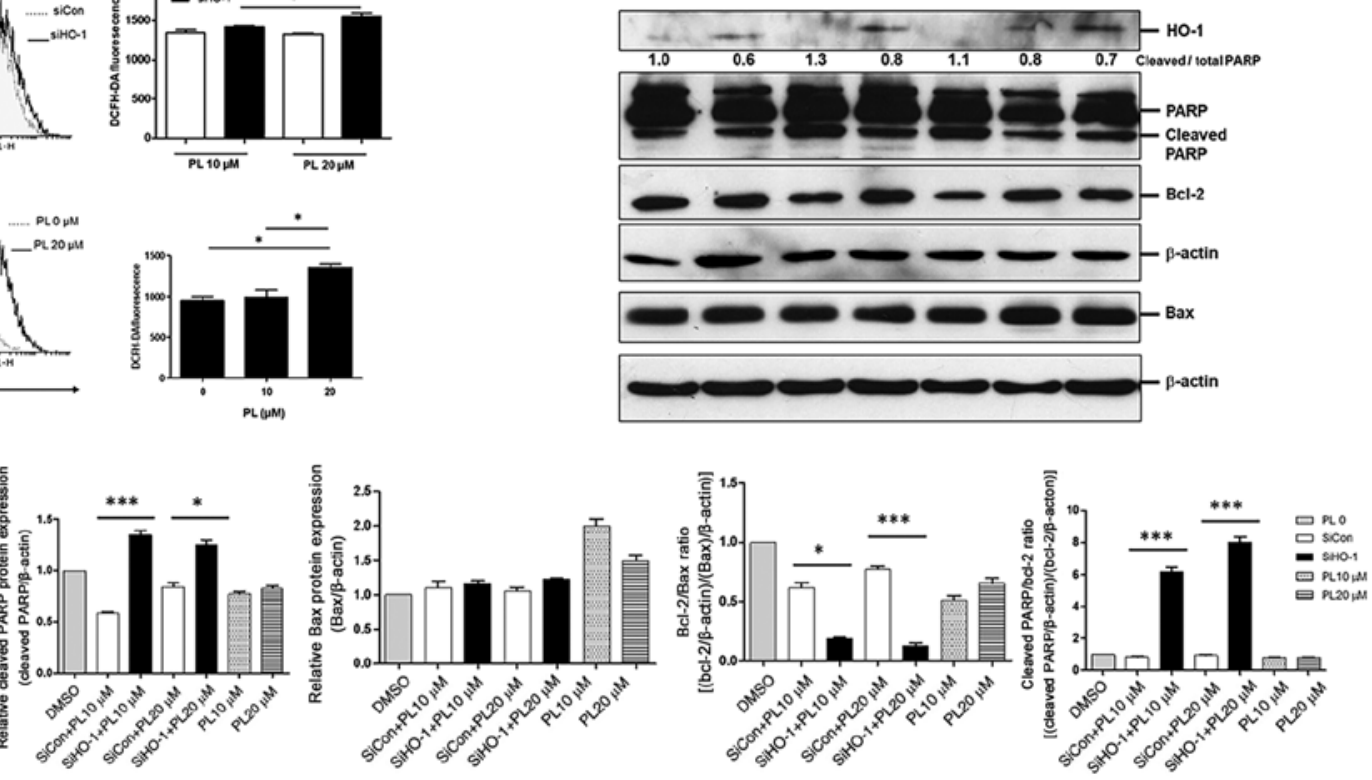

Figure 4. Knockdown of HO-1 by siRNA sensitizes cholangiocarcinoma cell lines to PL. KKU-100 and KKU-213A were transfected with siHO-1 or siCon for 24 and $48 \mathrm{~h}$. (A) The efficiency of siHO-1 knockdown at 24 and $48 \mathrm{~h}$ relative to siCon was determined using reverse transcription-quantitative PCR (B) Proliferative effect at $48 \mathrm{~h}$ and (C) the anti-proliferative effect of PL at various concentrations at $24 \mathrm{~h}$ were determined. (D) Reactive oxygen species accumulation at 3 (left graph) and $12 \mathrm{~h}$ (right graph). (E) Apoptotic and anti-apoptotic proteins at $12 \mathrm{~h}$ were determined and compared between HO-1-knockdown cells and controls. (F) Relative protein levels of cleaved PARP and Bcl-2, Bax and the Bcl-2/Bax ratio and cleaved PARP/Bcl-2 ratios were determined. Values are expressed as the mean \pm standard error of the mean of 3 independent experiments. ${ }^{*} \mathrm{P}<0.05$ and ${ }^{* * * *} \mathrm{P}<0.001$ vs. siCon. HO-1, heme oxygenase 1 ; siRNA, small interfering RNA; PL, piperlongumine; siHO-1, HO-1 siRNA; siCon, siRNA control (scrambled); PARP, poly(ADP-ribose) polymerase; DCFH-DA, 2',7'-dichlorodihydrofluorescein diacetate; OD564, optical density at $564 \mathrm{~nm}$.

A

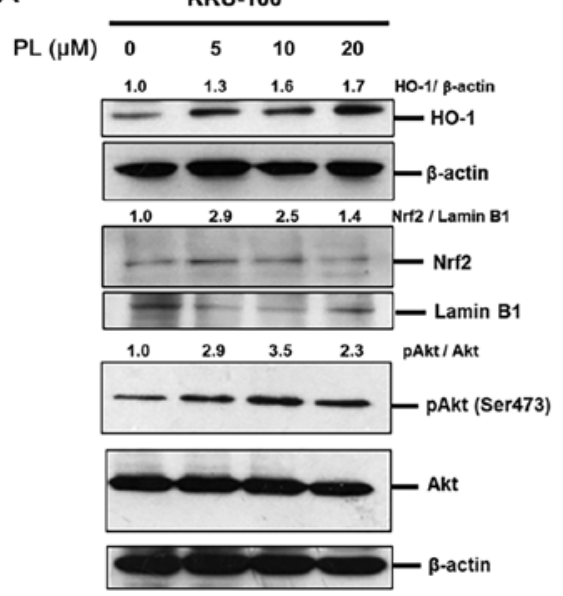

B

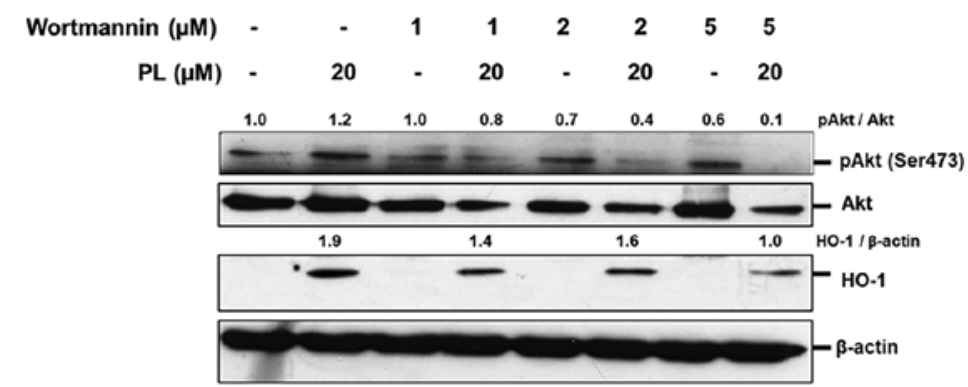

Figure 5. PL induces HO-1 expression via PI3K/Akt activation. (A) KKU-100 was treated with various concentrations of PL (0, 5, 10 or $20 \mu \mathrm{M})$ for $3 \mathrm{~h}$. Whole-cell lysates (for HO-1, Akt and $\beta$-actin) and nuclear extracts (for Nrf2 and Lamin B1) were analyzed via western blotting and probed with antibodies specific to HO-1, Akt, pAkt (Ser473), Nrf2 and $\beta$-actin. (B) The effects of the Akt signaling inhibitor wortmannin on PL-induced HO-1 expression were determined. KKU-100 was pre-treated with various concentrations of wortmannin $(1,2$ and $5 \mu \mathrm{M})$ for $2 \mathrm{~h}$ and treated with $20 \mu \mathrm{M}$ PL for an additional $24 \mathrm{~h}$. The expression of HO-1, Akt, pAkt and $\beta$-actin were determined via western blot analysis. Values are expressed as the mean of 2 independent experiments. PL, piperlongumine; pAkt, phosphorylated Akt; HO-1, heme oxygenase 1; Nrf2, nuclear factor erythroid 2-related factor 2. 
tumor growth via the induction of ROS accumulation and the activation of MAPKs (including JNK, ERK and p38) $(14,15)$ or the inhibition of the PI3K/Akt pathway $(35,36)$. Sensitivity to PL varies among different cancer cell lines, including breast cancer and CCA cell lines $(15,34)$. The present study aimed to investigate the underlying mechanisms of PL-induced HO-1 expression. Several previous studies have demonstrated that HO-1 has a powerful cytoprotective effect against various apoptotic insults in normal and cancer cells (19-23). The basal HO-1 expression levels of each type of cancer cell indicate its sensitivity to chemotherapeutic agents $(20,21)$. Similar observations were reported for CCA cells treated with PL, as cells with a low basal level of HO-1 expression were more sensitive to PL compared with those with high HO-1 expression (15). In addition, in the present study, HO-1 inducible expression was evident following 3 and $6 \mathrm{~h}$ of PL treatment in all CCA cell lines. This result indicated that induction of HO-1 expression serves a vital role in the early protection against the PL-induced reaction to oxidative stress. The results of the present study are consistent with those of studies on well-known chemotherapeutic agents (including gemcitabine and cisplatin), which mediate oxidative stress and lead to induced HO-1 expression in CCA and laryngeal squamous cell cancer $(20,23)$.

The PI3K/Akt pathway is involved in cellular survival, metastasis and drug resistance in various types of cancer including CCA, pancreatic and oral cancer (37-41). Development of acquired resistance to radiation, chemotherapy and/or targeted therapy has been revealed to be associated with the induction of the PI3K/Akt pathway $(41,42)$. In addition, PI3K/Akt signaling is a key pathway for the activation of HO-1 through the Nrf2/Keap pathway. Certain studies have demonstrated that PL bears two electrophilic $\alpha, \beta$-unsaturated carbonyl groups that cause oxidation or covalent modification of cysteine residues within Keap1. PL directly binds to cysteine residues within Keap1 by thiol modification (34). This direct binding was observed to promote nuclear translocation of Nrf2 and subsequent upregulation of HO-1 expression $(34,40)$. Inhibition of this pathway has been proposed to increase the chemosensitivity of CCA $(38,43)$. The present study revealed that PL treatment resulted in Akt phosphorylation and $\mathrm{Nrf} 2$ activation, suggesting that $\mathrm{PL}$ induced HO-1 expression via $\mathrm{PI} 3 \mathrm{~K} / \mathrm{Akt} / \mathrm{Nrf} 2$ activation. However, these results are preliminary and should be confirmed in a future study. In contrast to PL-induced antioxidant defense in CCA, PL stimulated oxidative stress via ROS generation and induced CCA apoptosis through the activation of the ROS/JNK/ERK pathway (15). These results indicated that in an oxidative stress environment, i.e. that caused by PL, CCA cells upregulate anti-oxidant defenses by inducing PI3K/Akt-mediated HO-1 expression and enhance the anti-apoptotic capacity in order to protect against PL-induced ROS generation. As Keap1 was not assessed in PL-induced Nrf2 activation and HO-1 expression in the present study, investigating the effect of PL on the Nrf2/Keap1 pathway in CCA is required for further study.

HO-1 is a powerful antioxidant enzyme in Nrf2-mediated cytoprotective responses and serves a role in the malignant transformation of cancer cells (10). High levels of HO-1 are associated with the progression of CCA and its therapeutic resistance $(20,21)$. Concurrently, suppression of HO-1 activity has been observed to enhance the chemosensitivity of cancer cells to various chemotherapeutic agents including cisplatin and gemcitabine $(20,23,44)$. The results of the present study demonstrated that inhibition of HO-1 activity using chemical inhibitors or specific siRNA to HO-1 increased the anti-tumor activity of PL against CCA cell lines through an increase in intracellular ROS accumulation and increased CCA cell death via upregulation of apoptotic proteins. In the present study, the increase of intracellular ROS was not significantly different between siCon KKU-100 cells treated with PL at 10 and $20 \mu \mathrm{M}$. This may be due to the insufficient concentration of $\mathrm{PL}$, as the $\mathrm{IC}_{50}$ value of PL in KKU-100 is $15.9 \mu \mathrm{M}$ at $24 \mathrm{~h}(15)$, or PL may have altered the activity of a redox-sensitive enzyme/transcription protein. In summary, the results of the present study suggest that induction of HO-1 expression may provide a significant antioxidant defense to PL treatment and the level of basal HO-1 expression indicates the efficiency of PL for treating CCA. It should be noted that the CCA cell lines used in the present study were established from tumors of patients with CCA with liver-fluke infections. Therefore, the results may not be generalizable to patients with non-liver fluke-associated CCAs. From a technical point, the experiments should ideally be performed three times. However, the determination of protein expression via western blot analysis was performed only twice. This limitation could influence the interpretation of the findings; thus, future studies are required to confirm.

In conclusion, in a PL-induced oxidative stress environment, PI3K/Akt-mediated HO-1 activation served an important role in antioxidant defenses, thereby protecting CCA from PL-induced apoptosis. The results demonstrated that suppression of $\mathrm{HO}-1$ resulted in increased intracellular ROS generation and CCA cell apoptosis induced by PL. The present results provide strong evidence that the mechanism of PL-induced HO-1 expression in CCA and inhibition of HO-1 may be a potential strategy for increasing the chemosensitivity of CCA to PL.

\section{Acknowledgements}

The authors would like to thank Mr. Bryan Roderick Hamman (Aegis of the Khon Kaen University Publication Clinic, Khon Kaen, Thailand) for his assistance in translating the manuscript into English.

\section{Funding}

The present study was supported by the Young Research Grants, Thailand Research Fund (grant no. MRG6080055 to CT).

\section{Availability of data and materials}

All data generated or analyzed during the present study are included in this published article.

\section{Authors' contributions}

CT and SW conceived and designed the present study. CT performed the experiments. CT, KT and SW analyzed the data. All authors read and approved the final manuscript. 


\section{Ethics approval and consent to participate}

Not applicable.

\section{Patient consent for publication}

Not applicable.

\section{Competing interests}

The authors declare that they have no competing interests.

\section{References}

1. Khan SA, Davidson BR, Goldin RD, Heaton N, Karani J, Pereira SP, Rosenberg WM, Tait P, Taylor-Robinson SD, Thillainayagam AV, et al; British Society of Gastroenterology: Guidelines for the diagnosis and treatment of cholangiocarcinoma: An update. Gut 61: 1657-1669, 2012.

2. Bhudhisawasdi V, Talabnin C, Pugkhem A, Khuntikeo N, Seow OT, Chur-in S, Pairojkul C and Wongkham S: Evaluation of postoperative adjuvant chemotherapy for intrahepatic cholangiocarcinoma patients undergoing R1 and R2 resections. Asian Pac J Cancer Prev 13: 169-174, 2012.

3. Dhanasekaran R, Hemming AW, Zendejas I, George T, Nelson DR, Soldevila-Pico C, Firpi RJ, Morelli G, Clark V and Cabrera R: Treatment outcomes and prognostic factors of intrahepatic cholangiocarcinoma. Oncol Rep 29: 1259-1267, 2013.

4. Nagino M, Ebata T, Yokoyama Y, Igami T, Sugawara G Takahashi Y and Nimura Y: Evolution of surgical treatment for perihilar cholangiocarcinoma: A single-center 34-year review of 574 consecutive resections. Ann Surg 258: 129-140, 2013.

5. Nagorney DM, Donohue JH, Farnell MB, Schleck CD and Ilstrup DM: Outcomes after curative resections of cholangiocarcinoma. Arch Surg 128: 871-879, 1993.

6 . Thongprasert $S$ : The role of chemotherapy in cholangiocarcinoma. Ann Oncol 16 (Suppl 2): ii93-ii96, 2005.

7. Fodale V, Pierobon M, Liotta L and Petricoin E: Mechanism of cell adaptation: When and how do cancer cells develop chemoresistance? Cancer J 17: 89-95, 2011.

8. Holohan C, Van Schaeybroeck S, Longley DB and Johnston PG Cancer drug resistance: an evolving paradigm. Nat Rev Cancer 13: 714-726, 2013.

9. Pelicano H, Carney D and Huang P: ROS stress in cancer cells and therapeutic implications. Drug Resist Updat 7: 97-110, 2004

10. Gorrini C, Harris IS and Mak TW: Modulation of oxidative stress as an anticancer strategy. Nat Rev Drug Discov 12: 931-947, 2013.

11. Guan J, Lo M, Dockery P, Mahon S, Karp CM, Buckley AR Lam S, Gout PW and Wang YZ: The xc-cystine/glutamate antiporter as a potential therapeutic target for small-cell lung cancer: Use of sulfasalazine. Cancer Chemother Pharmacol 64: 463-472, 2009.

12. Montero AJ, Diaz-Montero CM, Deutsch YE, Hurley J, Koniaris LG, Rumboldt T, Yasir S, Jorda M, Garret-Mayer E, Avisar E, et al: Phase 2 study of neoadjuvant treatment with NOV-002 in combination with doxorubicin and cyclophosphamide followed by docetaxel in patients with HER-2 negative clinical stage II-IIIc breast cancer. Breast Cancer Res Treat 132: 215-223, 2012.

13. Trachootham D, Alexandre J and Huang P: Targeting cancer cells by ROS-mediated mechanisms: A radical therapeutic approach? Nat Rev Drug Discov 8: 579-591, 2009.

14. Liu JM, Pan F, Li L, Liu OR, Chen Y, Xiong XX, Cheng K, Yu SB, Shi Z, Yu CH, et al: Piperlongumine selectively kills glioblastoma multiforme cells via reactive oxygen species accumulation dependent JNK and p38 activation. Biochem Biophys Res Commun 437: 87-93, 2013.

15. Thongsom S, Suginta W, Lee KJ, Choe H and Talabnin C: Piperlongumine induces $\mathrm{G} 2 / \mathrm{M}$ phase arrest and apoptosis in cholangiocarcinoma cells through the ROS-JNK-ERK signaling pathway. Apoptosis 22: 1473-1484, 2017.

16. Raj L, Ide T, Gurkar AU, Foley M, Schenone M, Li X, Tolliday NJ, Golub TR, Carr SA, Shamji AF, et al: Selective killing of cancer cells by a small molecule targeting the stress response to ROS. Nature 475: 231-234, 2011.
17. Maines MD and Abrahamsson PA: Expression of heme oxygenase-1 (HSP32) in human prostate: normal, hyperplastic, and tumor tissue distribution. Urology 47: 727-733, 1996.

18. Goodman AI, Choudhury M, da Silva JL, Schwartzman ML and Abraham NG: Overexpression of the heme oxygenase gene in renal cell carcinoma. Proc Soc Exp Biol Med 214: 54-61, 1997.

19. Yin H, Fang J, Liao L, Maeda H and Su Q: Upregulation of heme oxygenase-1 in colorectal cancer patients with increased circulation carbon monoxide levels, potentially affects chemotherapeutic sensitivity. BMC Cancer 14: 436, 2014.

20. Kongpetch S, Kukongviriyapan V, Prawan A, Senggunprai L, Kukongviriyapan U and Buranrat B: Crucial role of heme oxygenase-1 on the sensitivity of cholangiocarcinoma cells to chemotherapeutic agents. PLoS One 7: e34994, 2012.

21. Kongpetch S, Puapairoj A, Ong CK, Senggunprai L, Prawan A, Kukongviriyapan U, Chan-On W, Siew EY, Khuntikeo N, Teh BT, et al: Haem oxygenase 1 expression is associated with prognosis in cholangiocarcinoma patients and with drug sensitivity in xenografted mice. Cell Prolif 49: 90-101, 2016.

22. Furfaro AL, Piras S, Passalacqua M, Domenicotti C, Parodi A, Fenoglio D, Pronzato MA, Marinari UM, Moretta L, Traverso N, et al: HO-1 up-regulation: A key point in high-risk neuroblastoma resistance to bortezomib. Biochim Biophys Acta 1842: 613-622, 2014

23. Lv X, Song DM, Niu YH and Wang BS: Inhibition of heme oxygenase- 1 enhances the chemosensitivity of laryngeal squamous cell cancer Hep-2 cells to cisplatin. Apoptosis 21: 489-501, 2016.

24. Sripa B, Leungwattanawanit S, Nitta T, Wongkham C, Bhudhisawasdi V,Puapairoj A,SripaCand Miwa M: Establishment and characterization of an opisthorchiasis-associated cholangiocarcinoma cell line (KKU-100). World J Gastroenterol 11: 3392-3397, 2005

25. Sripa B, Seubwai W, Vaeteewoottacharn K, Sawanyawisuth K, Silsirivanit A, Kaewkong W, Muisuk K, Dana P, Phoomak C, Lert-Itthiporn W, et al: Functional and genetic characterization of three cell lines derived from a single tumor of an Opisthorchis viverrini-associated cholangiocarcinoma patient. Hum Cell: Mar 23, 2020 (Epub ahead of print). doi: 10.1007/s13577-02000334-w.

26. Voigt W: Sulforhodamine B assay and chemosensitivity. Methods Mol Med 110: 39-48, 2005.

27. Livak KJ and Schmittgen TD: Analysis of relative gene expression data using real-time quantitative PCR and the 2(-Delta Delta C(T)) method. Methods 25: 402-408, 2001

28. Labbé RF, Vreman HJ and Stevenson DK: Zinc protoporphyrin: A metabolite with a mission. Clin Chem 45: 2060-2072, 1999.

29. Wang S, Avery JE, Hannafon BN, Lind SE and Ding WQ: Zinc protoporphyrin suppresses cancer cell viability through a heme oxygenase-1-independent mechanism: The involvement of the Wnt $/ \beta$-catenin signaling pathway. Biochem Pharmacol 85: 1611-1618, 2013.

30. Liu YS, Li HS, Qi DF, Zhang J, Jiang XC, Shi K, Zhang XJ and Zhang XH: Zinc protoporphyrin IX enhances chemotherapeutic response of hepatoma cells to cisplatin. World J Gastroenterol 20: 8572-8582, 2014

31. Cheng CC, Guan SS, Yang HJ, Chang CC, Luo TY, Chang J and Ho AS: Blocking heme oxygenase-1 by zinc protoporphyrin reduces tumor hypoxia-mediated VEGF release and inhibits tumor angiogenesis as a potential therapeutic agent against colorectal cancer. J Biomed Sci 23: 18, 2016.

32. Martin D, Rojo AI, Salinas M, Diaz R, Gallardo G, Alam J, Galarreta CM and Cuadrado A: Regulation of heme oxygenase-1 expression through the phosphatidylinositol 3-kinase/Akt pathway and the Nrf 2 transcription factor in response to the antioxidant phytochemical carnosol. J Biol Chem 279: 8919-8929, 2004.

33. Chen HH, Chen YT, Huang YW, Tsai HJ and Kuo CC: 4-Ketopinoresinol, a novel naturally occurring ARE activator, induces the Nrf2/HO-1 axis and protects against oxidative stress-induced cell injury via activation of PI3K/AKT signaling. Free Radic Biol Med 52: 1054-1066, 2012.

34. Lee HN, Jin HO, Park JA, Kim JH, Kim JY, Kim B, Kim W, Hong SE, Lee $\mathrm{YH}$, Chang $\mathrm{YH}$, et al: Heme oxygenase-1 determines the differential response of breast cancer and normal cells to piperlongumine. Mol Cells 38: 327-335, 2015.

35. Wang F, Mao Y, You Q, Hua D and Cai D: Piperlongumine induces apoptosis and autophagy in human lung cancer cells through inhibition of PI3K/Akt/mTOR pathway. Int J Immunopathol Pharmacol 28: 362-373, 2015. 
36. Zhou L, Li M, Yu X, Gao F and Li W: Repression of hexokinases II-mediated glycolysis contributes to piperlongumine-induced tumor suppression in non-small cell lung cancer cells. Int J Biol Sci 15: 826-837, 2019.

37. Yokoi K, Kobayashi A, Motoyama H, Kitazawa M, Shimizu A, Notake T, Yokoyama T, Matsumura T, Takeoka M and Miyagawa SI: Survival pathway of cholangiocarcinoma via AKT/mTOR signaling to escape RAF/MEK/ERK pathway inhibition by sorafenib. Oncol Rep 39: 843-850, 2018.

38. Yoon H, Min JK, Lee JW, Kim DG and Hong HJ: Acquisition of chemoresistance in intrahepatic cholangiocarcinoma cells by activation of AKT and extracellular signal-regulated kinase (ERK)1/2. Biochem Biophys Res Commun 405: 333-337 2011.

39. Yothaisong $S$, Dokduang $H$, Techasen A, Namwat N, Yongvanit $P$, Bhudhisawasdi V, Puapairoj A, Riggins GJ and Loilome W: Increased activation of PI3K/AKT signaling pathway is associated with cholangiocarcinoma metastasis and $\mathrm{PI} 3 \mathrm{~K} / \mathrm{mTOR}$ inhibition presents a possible therapeutic strategy. Tumour Biol 34 3637-3648, 2013.
40. Chen HH, Chen YT and Huang YW: 4-Ketopinoresinol, a novel naturally occurring ARE activator, induces the Nrf2/HO-1 axis and protects against oxidative stress-induced cell injury via activation of PI3K/AKT signaling. Free Radic Biol Med 52: 1054-1066, 2012.

41. Arlt A, Gehrz A, Muerkoster S, Vorndamm J, Kruse ML, Folsch UR and Schäfer H: Role of NF-kappaB and Akt/PI3K in the resistance of pancreatic carcinoma cell lines against gemcitabine-induced cell death. Oncogene 22: 3243-3251, 2003.

42. Huang WC and Hung MC: Induction of Akt activity by chemotherapy confers acquired resistance. J Formos Med Assoc 108: 180-194, 2009.

43. Leelawat K, Narong S, Udomchaiprasertkul W, Leelawat S and Tungpradubkul S: Inhibition of PI3K increases oxaliplatin sensitivity in cholangiocarcinoma cells. Cancer Cell Int 9: 3, 2009.

44. Berberat PO, Dambrauskas Z, Gulbinas A, Giese TG, Kunzli B, Autschbach F, Meuer S, Büchler MW and Friess H: Inhibition of heme oxygenase-1 increases responsiveness of pancreatic cancer cells to anticancer treatment. Clin Cancer Res 11:3790-3798, 2005. 